\title{
Self-cleaning on a higher order mode in Ytterbium-doped multimode fiber with parabolic profile
}

\author{
Alioune Niang ${ }^{1 *}$, Vincent Couderc ${ }^{2}$, Alessandro Tonello ${ }^{2}$, Katarzyna Krupa ${ }^{1}$, Mesay Addisu ${ }^{1}$, Raphaël \\ Jauberteau $^{1,2}$, Marc Fabert ${ }^{2}$, Daniele Modotto ${ }^{1}$, and Stefan Wabnitz ${ }^{3}$ \\ ${ }^{1}$ Dipartimento di Ingegneria dell'Informazione, Università di Brescia, via Branze 38, 25123, Brescia, Italy \\ ${ }^{2}$ Université de Limoges, XLIM, UMR CNRS 7252, 123 Avenue A. Thomas, 87060 Limoges, France \\ ${ }^{3}$ DIET, Sapienza University of Rome, Via Eudossiana 18, 00184 Rome, Italy \\ Authore-mail address: alioune.niang@unibs.it
}

\begin{abstract}
We experimentally demonstrate polarization-dependent Kerr spatial beam self-cleaning into the LP11 mode of an Ytterbium-doped multimode optical fiber with parabolic gain and refractive index profiles. (C) 2019 The Author(s)

OCIS codes: (060.4370) Nonlinear optics, fibers; (190.3270) Kerr effect; (060.2320) Fiber optics Amplifiers and oscillators; (190.5940) Self-action effects
\end{abstract}

Although single mode fibers are well known for enabling efficient and spatially coherent supercontinuum sources, and for providing a convenient platform in the study of wide range of nonlinear phenomena, their small mode area largely prevents their use in the context of high power beam delivery and fiber laser applications. Due to their wide core area, multimode optical fibers (MMFs) are routinely used for high-power fiber lasers and industrial or biomedical beam delivery. However, the inherent degradation of the spatial beam quality, combined with dispersive pulse spreading in MMFs, imposes a precise and adaptive control of the input field via complex wave shaping techniques. It has been recently proposed by Wright et al. to exploit the fiber nonlinear response as a versatile means to control the spatiotemporal nonlinear dynamics of beams and pulses in graded-index MMFs [1]. A particularly impressive demonstration of such approach is provided by spatial beam self-cleaning [2,3], whereby above a certain threshold power the Kerr nonlinearity reshapes of output multimode intensity pattern into a robust and bell-shaped beam, close to fundamental LP01 mode. To date, Kerr self-cleaning has only been observed in graded-index (GRIN) MMFs, and not in step-index MMFs. For the use of nonlinear beam control in multimode fiber amplifiers and lasers, it is important to consider the presence of beam self-cleaning in active, rare-earth doped MMFs. Surprisingly, Guenard et al. have demonstrated that the presence of gain in a pumped $\mathrm{Yb}$-doped step-index MMF leads to efficient spatial self-cleaning into the fundamental LP01 mode [4,5]. The possibility of achieving self-cleaning in a GRIN active MMF remains an open issue, since these fibers are not commercially available.

In this work, we reveal the striking result that stable self-cleaning in a large core area GRIN Yb-doped MMF occurs into higher order (LP11) mode of the fiber. Moreover, Kerr self-cleaning exhibits a significant nonlinear polarization dependence.

We designed the Yb-doped MMF with both parabolic index and doping profiles. The fiber has been custom fabricated by the Fiber Optics Research Institute (FORC) in Moscow. The experimental setup to study self-cleaning in a $1.5 \mathrm{~m}$ long $\mathrm{Yb}$-doped GRIN MMF is depicted in Fig.1A. The fiber (with core $100 \mu \mathrm{m}$ core diameter, and $350 \mu \mathrm{m}$ x $350 \mu \mathrm{m}$ cladding) parabolic refractive index profile is shown in the inset of Fig. $1 \mathrm{~A}$; its absorption was $150 \mathrm{~dB} / \mathrm{m}$ at $940 \mathrm{~nm}$ and $2 \mathrm{~dB} / \mathrm{m}$ at the seed wavelength of $1064 \mathrm{~nm}$, where it allows for the propagation of thousands of modes.

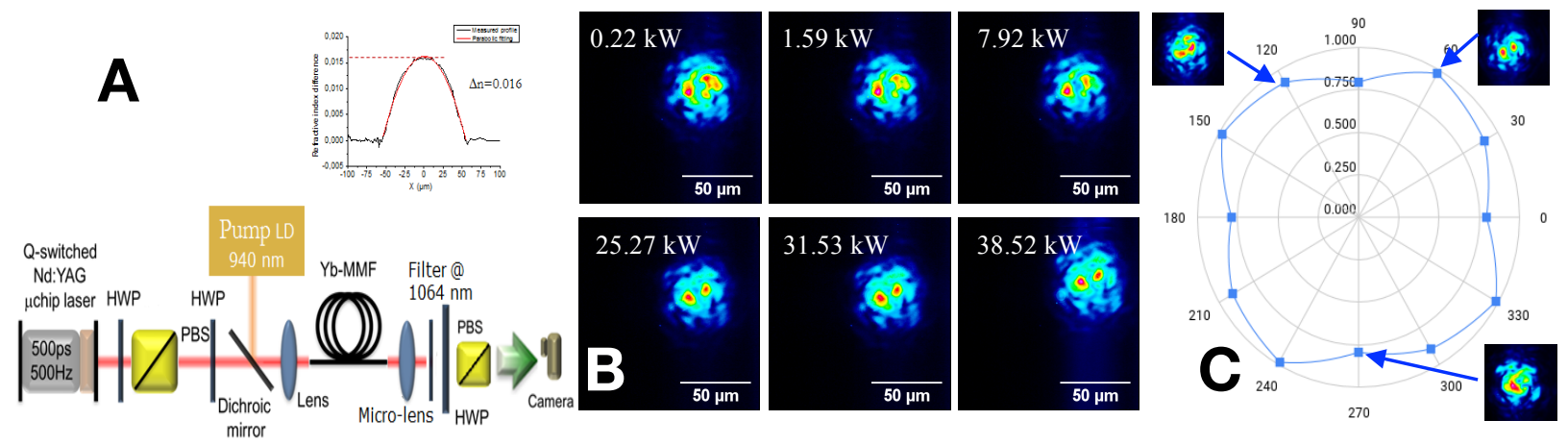

Fig. 1A Schematic of experimental setup to couple a microchip laser at $1064 \mathrm{~nm}$ (seed) and a pump laser diode (LD) into the Yb-doped MMF with core GRIN index profile as in the inset; B) Near-field spatial distribution of output beam vs. input peak power. Here the pump laser was turned off: C) Normalized output power and output intensity pattern for a full rotation of a half-wave plate (HWP) at the fiber output, followed by a polarizing beam splitter (PBS), for an input peak power fixed at $31.53 \mathrm{~kW}$. 
First, we studied the power dependence of the output spatial beam distribution of the Yb-doped GRIN MMF in the passive regime (i.e., without a pump laser). The initial diameter of the $1064 \mathrm{~nm}$ beam was optimized, in order to reduce number of excited modes in the fiber core. The input peak power was gradually increased from $200 \mathrm{~W}$ up to $40 \mathrm{~kW}$, and self-cleaning appeared with an output threshold power of $4.4 \mathrm{~kW}$. As shown in Fig. 1B, at the relatively low input powers from $0.22 \mathrm{~kW}$ up to $7.92 \mathrm{~kW}$, light at the fiber output exhibits a speckled spatial pattern. When the input peak power grows well above $7.92 \mathrm{~kW}$ up to $38.52 \mathrm{~kW}$, most of the injected power was transferred into the LP11 mode at the fiber output, see Fig.1B.

By turning an additional half-wave plate (HWP) followed by a polarizing beam splitter at the fiber output, we analyzed the state of polarization (SOP) at the output face of the fiber. Fig.1C illustrates the polarization dependence of the output beam power at $1064 \mathrm{~nm}$, normalized to its maximum value, for an input power fixed to $31.53 \mathrm{~kW}$. As can be seen, a self-cleaned output LP11 mode was not observed for all output SOPs. This is due to the fact that the self-cleaned LP11 mode has a dominant SOP, in contrast with the beam at low powers, which is depolarized.

Next, we studied the output spatial intensity distribution in the presence of both the signal and the pump laser. First, we measured the output fluorescence by using a spectral filter (with $10 \mathrm{~nm}$ bandwidth) at the seed wavelength, in order to block any residual pump light. We observed that the output fluorescence increases linearly with the pump power, and that fluorescence of the Yb-MMF at $1064 \mathrm{~nm}$ has a parabolic intensity profile. Next, we fixed the input peak power of the seed to $550 \mathrm{~W}$, with the pump laser switched off. Fig. 2A shows the corresponding output beam pattern profile. Finally, we turned on the pump laser by gradually increasing the LD power to provide gain (G) to the fiber. From Fig. 2A(b-g), it can be observed that by increasing the gain, self-cleaning of LP11 mode is obtained for gain values between $\mathrm{G}=3$ and $\mathrm{G}=21$ (at the maximum pump power). Self-cleaning appears above $1.65 \mathrm{~kW}$ of output power $(\mathrm{G}=3)$. This means that the presence of gain permits a $70 \%$ reduction of the self-cleaning threshold (in terms of output power) with respect to the passive configuration (where $4.4 \mathrm{~kW}$ of output power was needed). In the case of the self-cleaning into the fundamental mode with a step-index Yb-doped MMF, the gain-induced threshold reduction in $3 \mathrm{~m}$ of $\mathrm{Yb}-\mathrm{MMF}$ was more limited [4]. Fig. 2B shows a strong output beam pattern dependence its SOP.
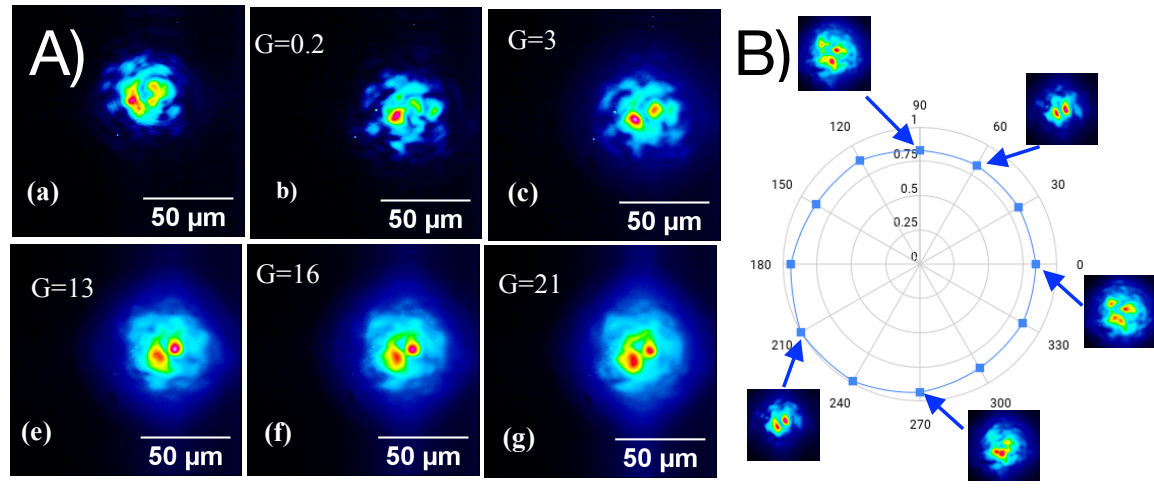

Fig. 2. By fixing the signal (seed) input peak power at $550 \mathrm{~W}$ : A) Near -field beam profile of the output beam vs. gain G (b-g). B) Normalized output power and output intensity pattern for a full output HWP rotation, and $\mathrm{G}=21$.

To conclude, we have experimentally shown that Kerr beam self-cleaning in a Yb-doped MMF can stably reshape the output beam pattern into the LP11 mode. Moreover, we observed that the output self-cleaned beam emerges essentially polarized. We anticipate that nonlinear effects in active MMFs will be of great relevance in the design of new multimode fiber lasers with controllable spatiotemporal properties.

We acknowledge support from: European Research Council (grant No. 740355); Marie-Skłodowska-Curie program (grant No. 713694).

\section{References}

[1] L.G. Wright, et al., "Controllable spatiotemporal nonlinear effects in multimode fibres," Nature Photonics 9, 306-310 (2015).

[2] Z. Liu, et al., "Kerr self-cleaning of femtosecond-pulsed beams in graded-index multimode fiber," Opt. Lett. 41, 3675-3678 (2016).

[3] K. Krupa, et al., "Spatial beam self-cleaning in multimode fibers," Nature Photonics 11, 237-241 (2017).

[4] R. Guenard, et al., "Kerr self-cleaning of pulsed beam in an ytterbium doped multimode fiber," Optics Express 25,4783-92, (2017).

[5] R. Guenard, et al., "Nonlinear beam self-cleaning in a coupled cavity composite laser based on multimode fiber," Optics Express 25, 22219-28, (2017). 